\title{
Oclusão artério-venosa da retina após bloqueio retrobulbar - Relato de dois casos
}

\author{
Combined central retinal vein and artery occlusion after retrobulbar anesthesia - \\ Report of two cases
}

\author{
Rogil José de Almeida Torres ${ }^{1}$ \\ Andréa Luchini ${ }^{2}$ \\ Wilma Weis ${ }^{3}$ \\ Paulo Roberto Frecceiro ${ }^{4}$ \\ Marcelo Casella ${ }^{5}$
}

Ex-residente e Ex-fellow de Retina e Vítreo do Instituto Penido Burnier. Aluno do Master Business of Administration - Instituto da Visão - da Universidade Federal de São Paulo - UNIFESP. São Paulo (SP).

${ }^{2}$ Médico do Centro Oftalmológico de Curitiba (PR).

${ }^{3}$ Médico do Centro Oftalmológico de Curitiba (PR).

${ }^{4}$ Médico do Centro Oftalmológico de Curitiba (PR).

Professor Adjunto Doutor em Oftalmologia pela Univer-

sidade Estadual de Londrina. Londrina (PR).

Endereço para correspondência: Rogil José de Almeida Torres - Praça Ruy Barbosa, 827/305 - Curitiba (PR) CEP 80010-030

E-mail: rjat@terra.com.br

Recebido para publicação em 08.09.2004 Aprovação em 15.12.2004

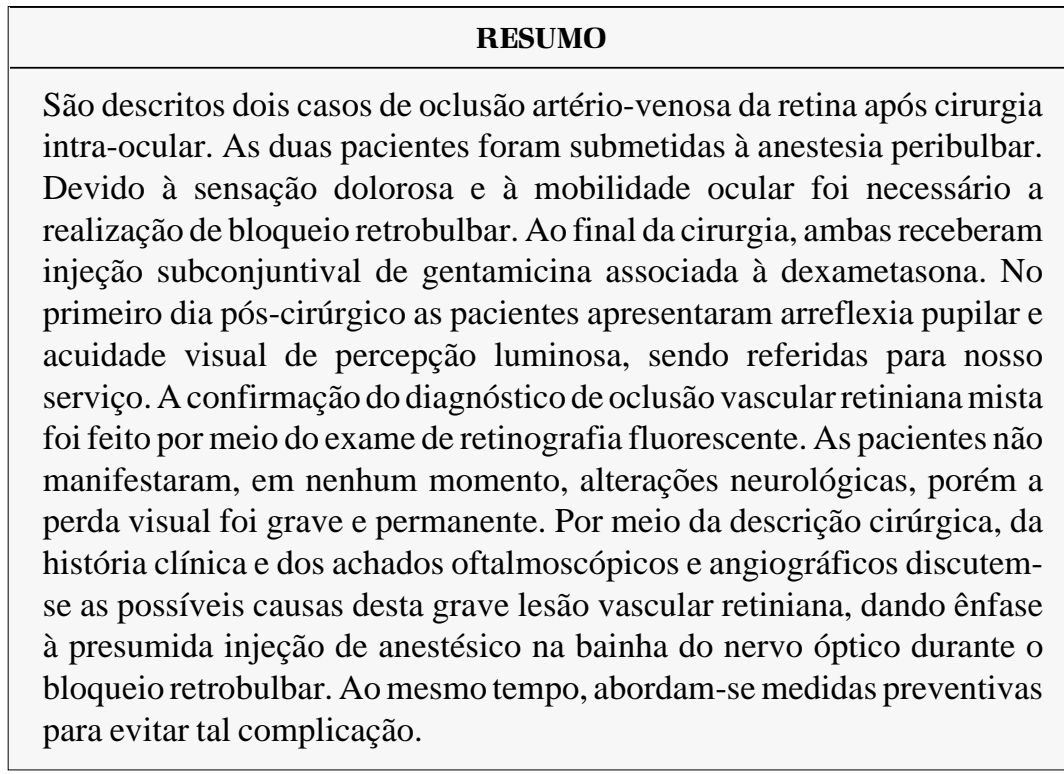

Descritores: Oclusão da artéria retiniana/etiologia; Anestesia local/efeitos adversos; Bloqueio nervoso; Procedimentos cirúrgicos oftalmológicos; Acuidade visual; Relatos de casos [Tipo de publicação]

\section{INTRODUC̄̃̃O}

A ocorrência de oclusão combinada de artéria e veia central da retina após cirurgia intra-ocular é rara ${ }^{(1-4)}$. Tal lesão vascular pode estar associada ao bloqueio retrobulbar. Este tipo de bloqueio foi o principal método para se obter acinesia e anestesia nas cirurgias oculares. Devido às complicações ${ }^{(5)}$, outras técnicas anestésicas menos invasivas e consequientemente menos traumáticas têm sido adotadas de rotina nas cirurgias intra-oculares, principalmente nas cirurgias de catarata. Entre os procedimentos alternativos destacamos a anestesia peribulbar e a anestesia tópica. O objetivo deste trabalho é apresentar dois casos de oclusão combinada da artéria e veia central da retina após presumida injeção de anestésico na bainha do nervo óptico. Outras possíveis etiologias são lembradas e discutidas como: compressão do nervo óptico provocada pelo hematoma retrobulbar, perfuração ocular provocada pela agulha utilizada no bloqueio anestésico e injeção inadvertida de gentamicina intra-ocular.

\section{Caso 1}

Paciente do sexo feminino, branca, 74 anos de idade, referia baixa grada- 
tiva da visão de olho direito. Ausência de doenças sistêmicas. Negava etilismo, tabagismo e uso de anticoagulante.

Ao exame oftalmológico constatou-se acuidade visual de OD igual 0,1. Reflexo pupilar aferente normal. A biomicroscopia demonstrou catarata nuclear. $\mathrm{O}$ exame de oftalmoscopia binocular indireta não identificou anormalidades do fundo de olho.

No dia 16/04/97 foi submetida a facectomia com implante de LIO. Inicialmente foi realizada anestesia peribulbar, utilizando uma mistura de $4 \mathrm{ml}$ de lidocaína $2 \%, 6 \mathrm{ml}$ de bupivacaína $0,75 \%$ sem adrenalina e hialuronidase $\left(15 \mathrm{UI}^{-\mathrm{ml}^{-1}}\right)$. Foram realizadas duas injeções peribulbares de $5 \mathrm{ml}$ cada uma, com agulha 30x7, uma na região ínfero-temporal no terço externo da reborda orbitária inferior e a outra súpero-temporal junto ao forame supra-orbitário, acompanhando a reborda orbitária superior. Após a injeção anestésica procedeu-se massagem sobre o globo ocular. Observou-se discreta proptose. Após 30 minutos, havia mobilidade do globo ocular e a paciente referia dor ao pinçar a conjuntiva. Devido à ineficiência da anestesia peribulbar foi realizado o bloqueio retrobulbar. Utilizou-se agulha $40 \times 7$, ponta romba, para injetar 3,5 $\mathrm{ml}$ de bupivacaína $0,75 \%$ sem adrenalina. Movimentos oculares não ocorreram enquanto a agulha estava no cone muscular. Procedeu-se aspiração após a injeção de anestésico, não havendo retorno sanguíneo. Após a realização da anestesia retrobulbar, colocou-se peso de mercúrio sobre o olho a ser operado. Não ocorreu proptose e não houve aumento da pressão intra-ocular. A injeção proporcionou completa anestesia e acinesia, possibilitando a extração da catarata extra-capsular com implante de lente intraocular, no sulco ciliar. Não houve evidência de toxicidade neurológica. Dados vitais mantiveram-se estáveis durante todo o procedimento. Ao final da cirurgia administrou-se injeção subconjuntival de gentamicina $20 \mathrm{mg}$ associada à dexametasona $12 \mathrm{mg}$.

No primeiro dia pós-operatório, 17/04/97, mostrava-se lúcida, sem alterações neurológicas. Apresentava arreflexia pupilar no olho operado. Acuidade visual de percepção luminosa e pressão intra-ocular de $23 \mathrm{mmHg}$. O exame de oftalmoscopia (Figura 1) demonstrou velamento de papila, focos hemorrágicos retinianos e pré-retinianos e palidez retiniana. Não havia solução de continuidade retiniana nem evidência de perfuração ocular. O exame de retinografia fluorescente (Figura 2) demonstrou predomínio da hipofluorescência determinada pela ausência de preenchimento da artéria central da retina e pelo bloqueio exercido pelos focos hemorrágicos retinianos. Preenchimento coroideano presente. A avaliação neurológica e o exame de ecodoppler de carótidas apresentavam-se dentro dos parâmetros de normalidade. Evoluiu com palidez da cabeça do nervo óptico e manteve acuidade visual de percepção luminosa.

\section{Caso 2}

Paciente do sexo feminino, branca, 66 anos. Apresentava HAS controlada. Negava etilismo, tabagismo e uso de anticoagulante. Referia ter sido submetida à cirurgia de catarata no olho direito, porém tal procedimento foi interrompido devido à ocorrência de ruptura de cápsula do cristalino. Apresen-

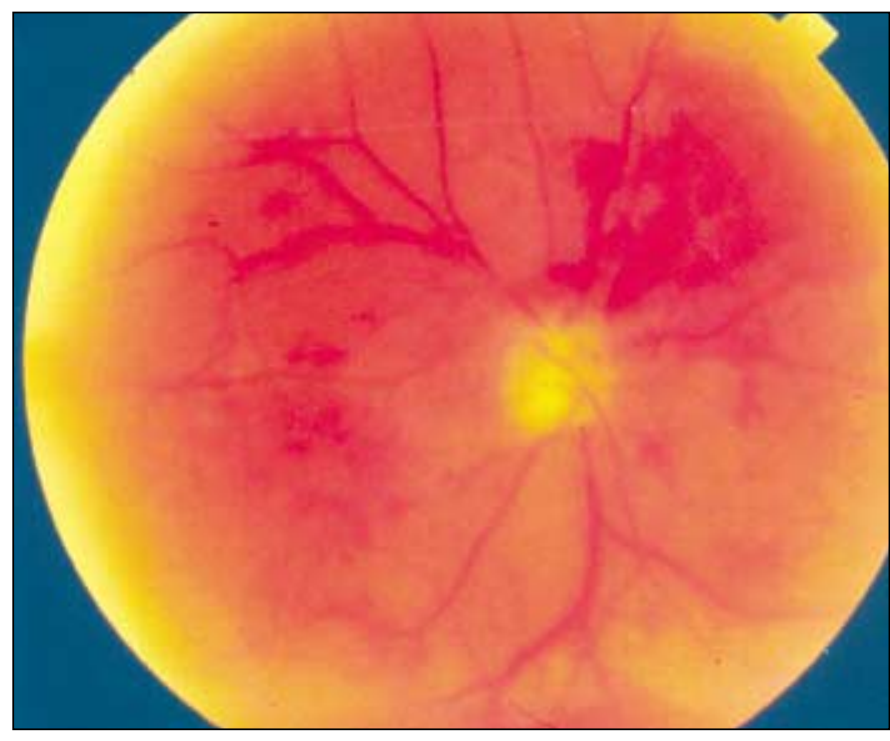

Figura 1 - Caso 1: Retinografia colorida demonstrando discreto velamento de papila. Hemorragias retinianas superficiais e profundas. Palidez retiniana

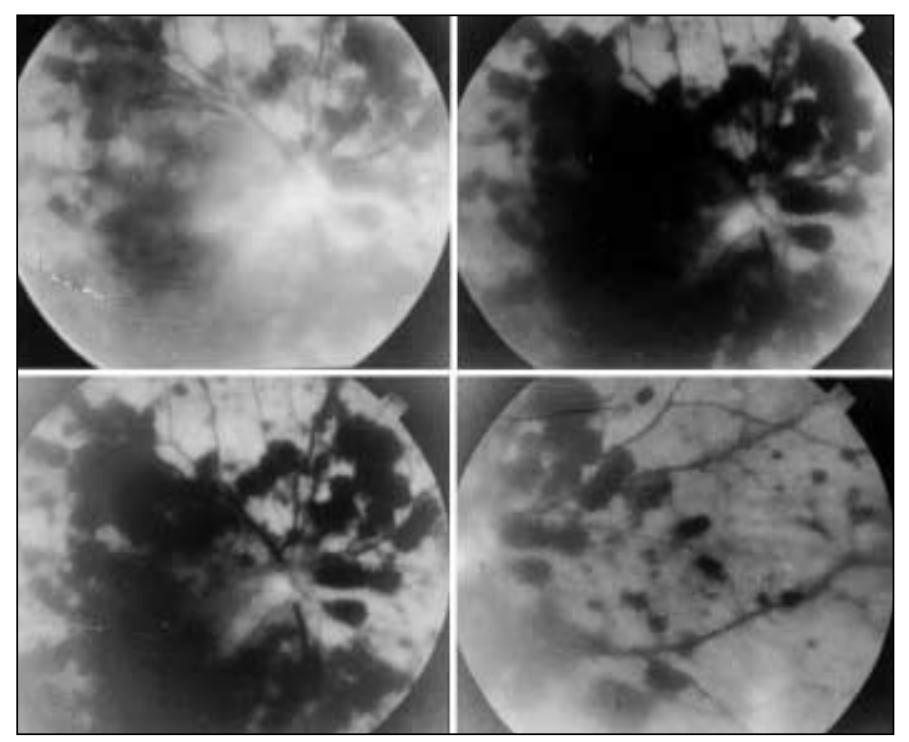

Figura 2 - Caso 1: Retinografia fluorescente demonstrando quadro característico da oclusão artério-venosa da retina. Focos hemorrágicos retinianos. Ausência de perfusão da artéria central da retina. Perfusão da circulação da coróide

tava acuidade visual de OD igual a 20/30 (com correção óptica). Reflexos fotomotores presentes e normais. O exame de biomiscroscopia demonstrou câmara anterior ampla e afácica. Pressão intra-ocular igual a $15 \mathrm{mmHg}$. O exame de oftalmoscopia binocular indireta revelou um fundo de olho normal.

No dia 19/07/2001 foi submetida à cirurgia de vitrectomia anterior com fixação escleral da LIO. Inicialmente foi realizada anestesia peribulbar, utilizando uma mistura de $4 \mathrm{ml}$ de lidocaína $2 \%, 6 \mathrm{ml}$ de bupivacaína $0,75 \%$ sem adrenalina e hialuronidase $\left(15\right.$ UI.ml $\left.{ }^{-1}\right)$. Foram realizadas duas injeções peribulbares 
de $5 \mathrm{ml}$ cada uma, com agulha 30x7, uma na região ínferotemporal no terço externo da reborda orbitária inferior e a outra súpero-temporal junto ao forame supra-orbitário, acompanhando a reborda orbitária superior. Após a injeção anestésica procedeu-se massagem sobre o globo ocular. Devido a persistente mobilidade do globo ocular optou-se por realizar o bloqueio retrobulbar. Utilizou-se agulha $40 \times 7$, ponta romba, para injetar 3,5 $\mathrm{ml}$ de bupivacaína $0,75 \%$ sem adrenalina retrobulbar. A injeção foi realizada no setor ínfero-temporal da órbita. Movimentos oculares não ocorreram enquanto a agulha encontrava-se no cone muscular. Procedeu-se aspiração após a injeção de anestésico, não havendo retorno sanguíneo. Após a realização da anestesia retrobulbar, colocou-se peso de mercúrio sobre o olho a ser operado. Não ocorreu proptose e não houve aumento da pressão intra-ocular. O bloqueio retrobulbar proporcionou oftalmoplegia e anestesia possibilitando a realização do procedimento cirúrgico, que ocorreu sem intercorrências. Não houve evidência de toxicidade neurológica e os dados vitais mantiveram-se estáveis durante o procedimento cirúrgico. Ao final da cirurgia foi injetado no espaço subconjuntival gentamicina $20 \mathrm{mg}$ associada à dexametasona $12 \mathrm{mg}$. No primeiro pós-operatório, dia 20/07/2001, a paciente não possuía queixas neurológicas e mostrava-se lúcida. Ao exame observou-se arreflexia pupilar do olho operado. Apresentava acuidade visual de percepção luminosa. A biomicroscopia demonstrava discreto edema de córnea e presença da lente intra-ocular no sulco ciliar. A pressão intra-ocular de 9 mmHg. O exame de oftalmoscopia demonstrou: velamento de papila, focos hemorrágicos intra-retinianos e pré-retinianos e palidez de retina (Figura 3). Não havia solução de continuidade retiniana nem evidência de perfuração ocular. O exame de angiografia demonstrou predomínio da hipofluorescência de fundo ocular, ocasionada pela ausência de perfusão da artéria central da retina e pelo bloqueio da fluorescência de fundo ocular provocado pelos focos hemorrágicos retinianos e préretinianos. Perfusão normal dos vasos da coróide (Figura 4). A avaliação neurológica e o ecodoppler de carótidas apresentavam-se normais. Evoluiu com palidez da cabeça do nervo óptico e manteve acuidade visual de percepção luminosa.

Observação: Os dados referentes à anestesia, ao procedimento cirúrgico e a evolução clínica foram adquiridos através de contato verbal com as respectivas equipes.

\section{DISCUSSÃO}

Injeção retrobulbar já foi um dos procedimentos mais comuns na oftalmologia, sendo usada para obter acinesia, anestesia ou para administrar drogas. Atualmente, este bloqueio é ainda utilizado nas intervenções oculares de longa duração, como as cirurgias vitreoretinianas. Mesmo que ocasionais, as ocorrências de complicações deste bloqueio nas cirurgias intra-oculares adquirem significação pelo alto número de cirurgias realizadas anualmente no mundo. Por este motivo, em

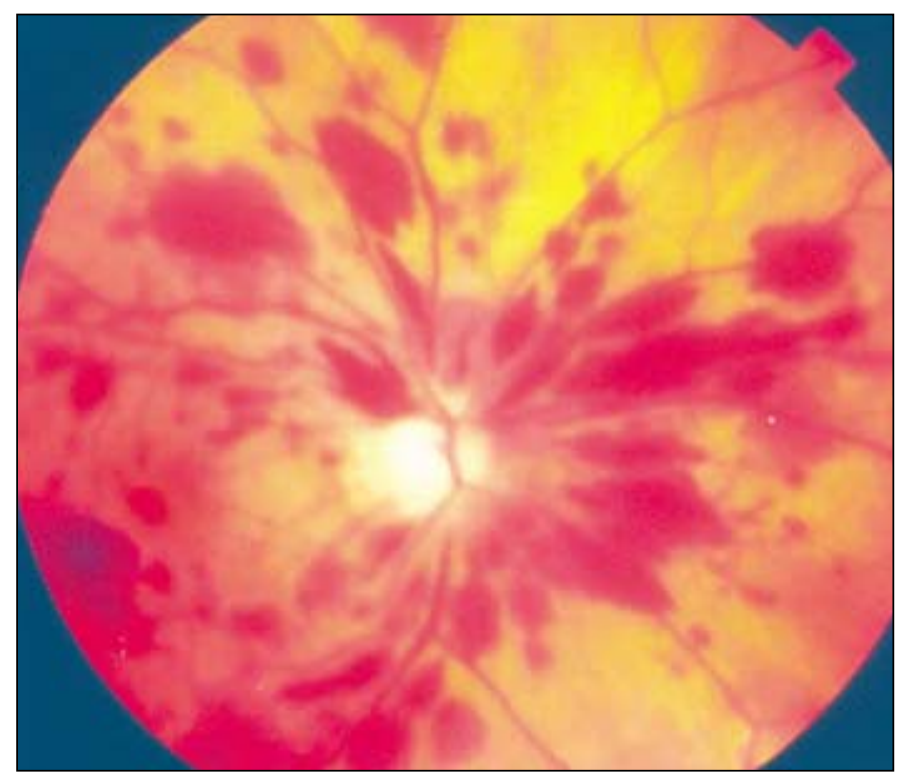

Figura 3- Caso 2: Retinografia colorida demonstrando discreto velamento de papila. Hemorragias retinianas superficiais e profundas. Palidez retiniana
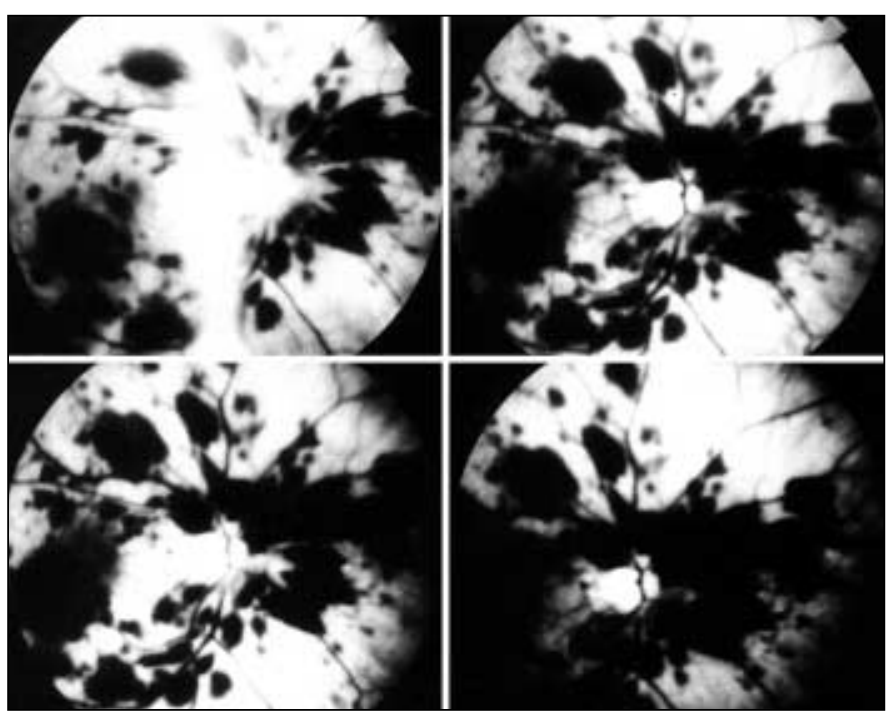

Figura 4 - Caso 2: Retinografia fluorescente demonstrando quadro característico da oclusão artério-venosa da retina. Focos hemorrágicos retinianos. Ausência de perfusão da artéria central da retina. Perfusão da circulação da coróide

procedimentos menos traumáticos e de curta duração, opta-se pelo bloqueio peribulbar ${ }^{(6)}$ ou até anestesia tópica ${ }^{(7)}$.

Durante várias décadas utilizou-se a técnica de Atkinson para a realização do bloqueio retrobulbar. O bloqueio era realizado com agulha de $35 \mathrm{~mm}$. O olho a ser bloqueado posicionava-se para cima e medialmente. Após inserir a agulha a $0,5 \mathrm{~cm}$ medialmente ao canto lateral, acima do rebordo orbitário inferior, direcionava-se sua extremidade para cima e medialmente com o intuito de entrar no cone muscular. Esta técnica coloca o nervo óptico em situação vulnerável ${ }^{(8)}$. 
As complicações do bloqueio retrobulbar são raras e usualmente ocorrem pelo trauma direto da agulha sobre as estruturas orbiculares ${ }^{(2)}$. $\mathrm{O}$ globo ocular pode ser afetado pela hemorragia retrobulbar, proptose, equimose, exposição corneana, perfuração escleral, embolização da artéria central da retina, obstrução dos vasos centrais da retina, amaurose ipsilateral e atrofia óptica ${ }^{(1-5,9-10)}$. Entre as complicações neurológicas, que se relacionam com a injeção de anestésico no espaço subaracnóideo do nervo óptico, destacam-se a amaurose contralateral, disfunção de nervos cranianos, oftalmoplegia, inconsciência, depressão respiratória e convulsão ${ }^{(11-13)}$.

Existem algumas hipóteses para explicar a ocorrência da oclusão vascular retiniana após anestesia retrobulbar. A primeira seria ocasionada pela hemorragia retrobulbar, que provoca aumento da pressão orbitária, superando a pressão sanguínea da artéria oftálmica ou artéria central da retina, levando à oclusão dos vasos retinianos ${ }^{(10)}$. Outra possibilidade relaciona-se com o trauma direto da agulha que levaria ao espasmo da artéria central da retina ${ }^{(5)}$. $\mathrm{O}$ efeito farmacológico do anestésico, induzindo à vasoconstrição, também poderia contribuir para o acidente vascular ${ }^{(5)}$. Alguns autores atribuíram a ocorrência da oclusão vascular retiniana à hemorragia na bainha do nervo óptico ${ }^{(1)}$. No entanto, foi demonstrado através da ecografia, tomografia computadorizada e à ressonância magnética que, na maioria dos casos descritos na literatura, a injeção foi realizada dentro da bainha do nervo óptico. Isto provoca aumento da pressão desta estrutura, comprometendo o fluxo da circulação venosa e arterial, ocasionando oclusão combinada da artéria e veia central da retina ${ }^{(1-2)}$.

As alterações oftalmológicas observadas, no pós-operatório imediato das duas pacientes, coincidem com as descritas na literatura, consistindo de dramática diminuição da acuidade visual, arreflexia pupilar e, ao exame de oftalmoscopia, presença de edema de papila, palidez retiniana, focos hemorrágicos intra e pré-retinianos dispersos no fundo ocular ${ }^{(1-4)}$. O exame de oftalmoscopia binocular indireta não demonstrou sinais de perfuração ocular, pois não havia ruptura de retina, hemorragia vítrea ou sinais de proliferação vítreo-retiniana ${ }^{(14)}$. Ao contatarmos as equipes de anestesia e de cirurgia para obter informações que nos levassem à causa desta complicação fomos informados que as pacientes haviam sido submetidas à anestesia peribulbar e que devido à ineficiência do bloqueio houve necessidade de realizar a anestesia retrobulbar. Também nos informaram que após a injeção retrobulbar não houve proptose e a pressão intra-ocular manteve-se estável durante o procedimento. Estes dados nos levaram a afastar, praticamente, a possibilidade de hematoma retrobulbar.

Também fomos informados que ao final do procedimento cirúrgico foi realizada, em ambas as pacientes, injeção subconjuntival de gentamicina associada à dexametasona, nos levando a pensar numa possível injeção intra-ocular acidental de antibiótico. Sabe-se que a injeção subconjuntival de aminoglicosídeo pode ocasionar toxicidade macular ${ }^{(15)}$. A gravidade aumenta significativamente quando o antibiótico ganha o espaço intra-ocular. Quadro de vasculopatia oclusiva isquêmica pode ser observado, com hemorragias retinianas, áreas de não perfusão capilar, edema de papila e finalmente a ocorrência de atrofia óptica e até glaucoma neovascular ${ }^{(16)}$. Existe normalmente uma nítida demarcação entre o segmento retiniano que teve contato com o antibiótico e a superfície retiniana que não teve ${ }^{(16)}$. Mesmo assim as alterações oftalmoscópicas e angiográficas assemelham-se às encontradas na infiltração intraneural de anestésico. Nas duas pacientes as alterações vasculares abrangeram toda a retina. Os focos hemorrágicos intra e pré-retinianos espalhavam-se por todo o fundo de olho caracterizando a oclusão venosa central. Ocorreu oclusão da artéria central da retina, não havendo perfusão de contraste no exame angiofluoresceinográfico nem nas fases tardias. Em ambos os casos as equipes cirúrgicas afirmaram que a gentamicina foi preparada após o término da cirurgia e injetada no espaço subconjuntival sob visualização microscópica. Este dado associado à ausência de sinais de perfuração ocular foram importantes para afastarmos a possibilidade de injeção intraocular do aminoglicosídeo.

Outra possibilidade aventada foi injeção intra-ocular de anestésico ${ }^{(17)}$. Apesar de provocar arreflexia e queda da acuidade visual no pós-operatório imediato, tal substância não provoca oclusão vascular retiniana e normalmente ocorre recuperação visual posteriormente. Além do mais não havia sinais de perfuração ocular.

Somando-se a quantidade de anestésico utilizado no bloqueio peribulbar e retrobulbar, o volume final administrado em ambas as pacientes excedeu a $13 \mathrm{ml}$. Esta quantidade é suficiente para ocasionar oclusão vascular retiniana em pacientes com severa doença vascular, como diabetes ${ }^{(9)}$. Inicialmente, as duas pacientes não apresentavam alterações vasculares sistêmicas. Sabe-se também que este tipo de obstrução é reversível ${ }^{(9)}$, oposto da oclusão vascular ocasionada pela gentamicina ${ }^{(16)}$ ou pela injeção intraneural de anestésico ${ }^{(4)}$, que são definitivas.

Após ponderarmos sobre todas as possibilidades, concluímos que esta grave alteração vascular retiniana foi ocasionada pela presumida injeção de anestésico na bainha do nervo óptico durante o bloqueio retrobulbar. Mesmo que possa demonstrar nervo óptico normal ${ }^{(3)}$, a realização de ecografia, tomografia computadorizada e/ou ressonância magnética da órbita seriam exames importantes para fazer o diagnóstico de injeção intraneural de anestésico ${ }^{(1)}$. Tais exames foram sugeridos, porém as pacientes foram submetidas somente à avaliação neurológica a qual mostrou-se normal. O que nos levou a concluir que houve a injeção de anestésico intraneural foi: $1^{0} \mathrm{o}$ tipo de agulha utilizada no bloqueio retro-bulbar, 40x7, era maior que a agulha adotada na técnica de Atkinson. O fato de possuir ponta romba não elimina a possibilidade de atingir e lesionar o nervo óptico ${ }^{(5)} ; 2^{\circ}$ oclusão vascular retiniana definitiva, abrangendo toda a superfície retiniana; $3^{\circ}$ ausência de sinais de perfuração ocular.

Não há duvida que o bloqueio retrobulbar oferece vantagens sobre a anestesia geral, pois é menos invasivo e conseqüentemente menos arriscado. Também pode ser utilizado quando a anestesia tópica ou peribulbar não obtiverem êxito. 
Sendo assim, este tipo de anestesia continua sendo usado em circunstâncias especiais. Por se tratar de inserção às escuras de uma agulha dentro de espaço ocupado por inúmeras estruturas vasculares e neurais, alguns cuidados devem ser tomados:

1을 Recomenda-se que o paciente mantenha os olhos na posição primária do olhar. No 1/3 final da introdução da agulha é conveniente solicitar que olhe para baixo e levemente para fora $^{(2,8,19)}$. Esta posição afasta o nervo óptico da extremidade da agulha.

$2^{\circ}$ Sugere-se também que a agulha seja romba ${ }^{(2,8)}$ e tenha comprimento menor ${ }^{(8)}$. Há estudos demonstrando que a agulha não deveria penetrar mais que $31 \mathrm{~mm}$ do rebordo orbitário ${ }^{(8)}$.

3 - Prestar atenção à resistência encontrada pela agulha. Se estiver aumentada convém puxá-la até encontrar a resistência normal $^{(18)}$.

$4^{0}$ Injetar lentamente o anestésico. Esta medida visa afastar as estruturas da ponta da agulha ${ }^{(2)}$.

$5^{\circ}$ Injetar pequenos volumes, de 2 a $3 \mathrm{ml}^{(2)}$.

60 Injetar enquanto a agulha é removida lentamente, para evitar que grande volume seja direcionado para um local ou estrutura $^{(2)}$.

$7^{0}$ Aconselha-se a realização de termo de ciência e consentimento antes da administração da injeção retrobulbar ${ }^{(2)}$.

A oclusão vascular retiniana após injeção retrobulbar provoca severa perda da função visual e assim sendo o diagnóstico precoce é muito importante. Intervenções como cantotomia e paracentese de câmara anterior, nos casos de hematoma retrobulbar $^{(9)}$, e descompressão da bainha do nervo óptico, nos casos de hematoma na bainha do nervo óptico ${ }^{(1)}$, podem ajudar a obter melhor resultado funcional.

Com pleno conhecimento anatômico da órbita e com os cuidados acima descritos dificilmente o anestesista terá complicações neste procedimento.

\section{ABSTRACT}

Two cases of combined central retinal artery and vein occlusion after intraocular surgery are described. Both patients were submitted to peribulbar anesthesia. Due to the painful sensation and ocular mobility retrobulbar anesthesia was necessary. At the end of the surgery both patients received a subconjunctival injection of gentamicin associated with dexamethasone. On the very first day after the surgery the two patients showed pupillary areflexia and visual acuity of luminous perception when they were referred to our service. The confirmation of the diagnosis of combined vascular occlusion of the retina was obtained by fluorescein angiography test. The two patients never showed, any neurological alteration. However visual loss was severe and permanent. By means of surgical description, clinical history, fundus photography and fluorescein angiography we are able to discuss the possible causes of this severe retinal vascular injury emphasizing the presumed anesthesic injection that was given into the optical nerve during retrobulbar anesthesia. At the same time we discuss preventive measures to avoid such problem.

Keywords: Retinal artery occlusion/etiology; Anesthesia, local/adverse effects; Nerve block; Ophthalmologic surgical procedures; Visual acuity; Case report [Publication type]

\section{REFERÊNCIAS}

1. Sullivan KL, Brown GC, Forman AR, Sergott RC, Flanagan JC. Retrobulbar anesthesia and retinal vascular obstruction. Ophthalmology. 1983;90(4):373-7.

2. Morgan CM, Schatz H, Vine AK, Cantrill HL, Davidorf FH, Gitter KA, Rudich R. Ocular complications associated with retrobulbar injections. Ophthalmology. 1988;95(5):660-5.

3. Mieler WF, Bennett SR, Platt LW, Koenig SB. Localized retinal detachment with combined central retinal artery and vein occlusion after retrobulbar anesthesia. Retina. 1990;10(4):278-83.

4. Giuffrè G, Vadala M, Manfrè L. Retrobulbar anesthesia complicated by combined central retinal vein and artery occlusion and massive vitreoretinal fibrosis. Retina. 1995;15(5):439-41.

5. Klein ML, Jampol LM, Condon PI, Rice TA, Serjeant GR. Central retinal artery occlusion without retrobulbar hemorrhage after retrobulbar anesthesia. Am J Ophthalmol. 1982;93(5):573-7.

6. David DB 2nd, Mandel MR. Peribulbar anesthesia. Ophthalmol Clin North Am. 1990;3(1):101-10.

7. Smith R. Cataract extraction without retrobulbar anaesthetic injection. Br J Ophthalmol. 1990;74(4):205-7.

8. Katsev DA, Drews RC, Rose BT. An anatomic study of retrobulbar needle path length. Ophthalmology. 1989;96(8):1221-4.

9. Cowley M, Campochiaro PA, Newman SA, Fogle JA. Retinal vascular occlusion without retrobulbar or optic nerve sheath hemorrhage after retrobulbar injection of lidocaine. Ophthalmic Surg. 1988;19(12):859-61.

10. Kraushar MF, Seelenfreund MH, Freilich DB. Central retinal artery closure during orbital hemorrhage from retrobulbar injection. Trans Am Acad Ophthalmol Otolaryngol. 1974;78(1):OP65-70.

11. Nicoll JM, Acharya PA, Ahlen K, Baguneid S, Edge KR. Central nervous system complications after 6000 retrobulbar blocks. Anesth Analg. 1987;66 (12):1298-302.

12. Wittpenn JR, Rapoza P, Sternberg P Jr, Kuwashima L, Saklad J, Patz A. Respiratory arrest following retrobulbar anesthesia. Ophthalmology. 1986;93 (7):867-70.

13. Antoszyk AN, Buckley EG. Contralateral decrease visual acuity and extraocular muscle palsies following retrobulbar anesthesia. Ophthalmology. 1986;93 (4):462-5.

14. Schneider ME, Milstein DE, Oyakawa RT, Ober RR, Campo R. Ocular perforation from a retrobulbar injection. Am J Ophthalmol. 1988;106(1):35-40.

15. Judson PH. Aminoglycoside macular toxicity after subconjunctival injection. Case report. Arch Ophthalmol. 1989;107(9):1282-3.

16. McDonald HR, Schatz H, Allen AW, Chenoweth RG, Cohen HB, Crawford JB, et al. Retinal toxicity secondary to intraocular gentamicin injection. Ophthalmology. 1986;93(7):871-7.

17. Lincoff H, Zweifach $\mathrm{P}$, Brodie S, Fuchs W, Gross S, Kornmehl E, et al. Intraocular injection of lidocaine. Ophthalmology. 1985;92(11):1587-91.

18. Brod RD. Transient central retinal artery occlusion and contralateral amaurosis after retrobulbar anesthetic injection. Ophthalmic Surg. 1989;20(9):643-6.

19. Friedberg HL, Kline OR Jr. Contralateral amaurosis after retrobulbar injection. Am J Ophthalmol. 1986;101(6):688-90. 